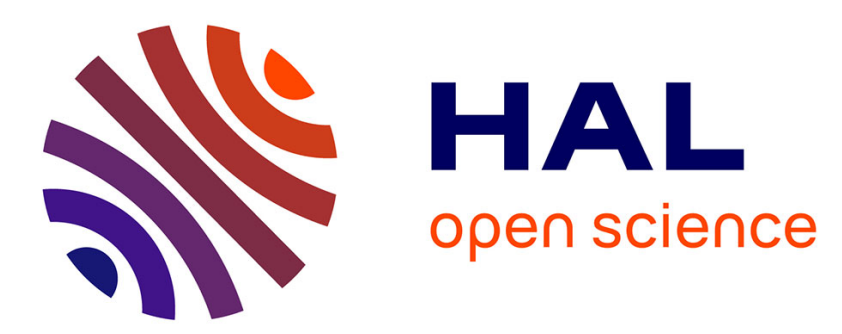

\title{
Prediction of the spectral reflectance of laser-generated color prints by combination of an optical model and learning methods
}

David Nébouy, Mathieu Hébert, Thierry Fournel, Nina Larina, Jean-Luc Lesur

\section{- To cite this version:}

David Nébouy, Mathieu Hébert, Thierry Fournel, Nina Larina, Jean-Luc Lesur. Prediction of the spectral reflectance of laser-generated color prints by combination of an optical model and learning methods. Journal of the Optical Society of America. A Optics, Image Science, and Vision, 2015, 32 (9), pp.1661-1671. 10.1364/JOSAA.32.001661 . hal-01185047

\section{HAL Id: hal-01185047 \\ https://hal.science/hal-01185047}

Submitted on 25 Aug 2015

HAL is a multi-disciplinary open access archive for the deposit and dissemination of scientific research documents, whether they are published or not. The documents may come from teaching and research institutions in France or abroad, or from public or private research centers.
L'archive ouverte pluridisciplinaire HAL, est destinée au dépôt et à la diffusion de documents scientifiques de niveau recherche, publiés ou non, émanant des établissements d'enseignement et de recherche français ou étrangers, des laboratoires publics ou privés. 


\title{
Prediction of the spectral reflectance of laser- generated color prints by combination of an optical model and learning methods
}

\author{
David Nebouy, ${ }^{1,2^{*}}$ Mathieu Hebert, ${ }^{1}$ Thierry Fournel, ${ }^{1}$ Nina Larina, ${ }^{2}$ Jean- \\ LUC LESUR ${ }^{2}$ \\ ${ }^{1}$ Université de Lyon, Université Jean Monnet de Saint Etienne, CNRS UMR 5516, Laboratoire Hubert Curien, F-42000 Saint Etienne, France \\ ${ }^{2}$ Gemalto SA Avenue du Pic de Bertagne, ZA de Gémenos, BP.100 F-13881 Gémenos Cedex, France \\ *Corresponding author: david.nebouy@univ-st-etienne.fr
}

Received XX Month XXXX; revised XX Month, XXXX; accepted XX Month XXXX; posted XX Month XXXX (Doc. ID XXXXX); published XX Month XXXX

Recent color printing technologies based on the principle of revealing colors on pre-functionalized achromatic supports by laser irradiation offer advanced functionalities, especially for security applications. However, for such technologies the color prediction is challenging, compared to classic ink-transfer printing systems. The spectral properties of the coloring materials modified by the lasers are not precisely known and may strongly vary, depending on the laser settings, in a nonlinear manner. We show in this study, through the example of the Color Laser Marking technology based on laser-bleaching of a mixture of pigments, that the combination of an adapted optical reflectance model and learning methods to get the model's parameters enables predicting the spectral reflectance of any printable color with rather good accuracy. Even though the pigment mixture is formulated from three colored pigments, an analysis of the dimensionality of the spectral space generated by CLM printing, thanks to a Principal Component Analysis decomposition, shows that at least four spectral primaries are needed for accurate spectral reflectance predictions. A polynomial interpolation is then used to relate RGB laser intensities with virtual coordinates of new basis vectors. By studying the influence of the number of calibration patches on the prediction accuracy, we can conclude that a reasonable number of 130 patches are enough to achieve good accuracy in this application.

OCIS codes: (120.5700) Reflection, (330.1690) Color, (100.2810) Halftone image reproduction, (350.6670) Surface photochemistry, (000.3860) Mathematical methods in physics.

\section{INTRODUCTION}

The usual definition for printing as application of inks on a support in order to transmit information [1] is still valid for most printing systems but is becoming too straight for certain printing processes under development. Owing to the progress in photonics and chemistry, the transfer of photo-active substances on the support and the revelation of information can be made in two separate steps. This is the case for the laser-induced color marking based on silver nanoparticles in crystalline matrices [2] where, for example, a clear $\mathrm{TiO}_{2} / \mathrm{Ag}$ layer is coated on the support, and then colored under laser irradiation [3]. The Color Laser Marking (CLM) technology studied in the present work is a second example dedicated to the card printing industry [4]]. The colored components, which form a sublayer of the card, are selectively bleached when exposed to laser irradiation, i.e., their spectral transmittances are modified. Since the color of every printable pixel can be modified, the system enables continuous-tone printing, i.e., without halftoning, similar to silver photograph printing. Compared to the Dye Diffusion Thermal Transfer (D2T2) technology which also enables continuous-tone printing on cards [1] , the interest in CLM is that printing can be processed after lamination of the card, and the production of color is made below the protection overlay to ensure security, whereas the D2T2 approach involves printing on the card surface and coating it with a protection patch: varnish or thin hot laminated overlay. Moreover, CLM is a more advanced technology that can add security features such as Kinegram ${ }^{\circledR}$ on the printing photo area and new ones specific to this technology. The structure of the card is more robust to attacks such as delamination of the card and ID photo replacing or modification thanks to irreversible color changes []․ Selectively bleaching pigments by laser sources as a means of secure printing is practically not investigated and is only found as a subject of patents [ $[\underline{6}, 7]$.

In the CLM technology, the photosensitive layer is a dark grey mixture involving three pigment-type colorants: cyan, magenta and yellow, coated onto a transparent polycarbonate film, which is then laminated together with the other polycarbonate films constituting the 
final card. Fig. 1 shows an area of white card where this mixture is deposited. Three lasers of different wavelengths are used to bleach the targeted pigments and "reveal" colors inside the polycarbonate card: a red laser bleaches the cyan pigments in the mixture, a green laser the magenta pigments, and a blue laser the yellow pigments. As the laser power increases, the dark dye mixture is more beached and the resulting color is brighter. The laser beam diameter is around 30 to 35 $\mu \mathrm{m}$ with a Gaussian irradiance profile and a variable input power encoded on 8 bits, which can therefore produce 256 irradiance values on the surface. The printing resolution is around $600-800 \mathrm{dpi}$ and, by tuning the power of the three lasers in each pixel, coloration of the surface can be achieved in an almost continuous way in contrast to halftoning in classic printing, as shown in Fig. 2.

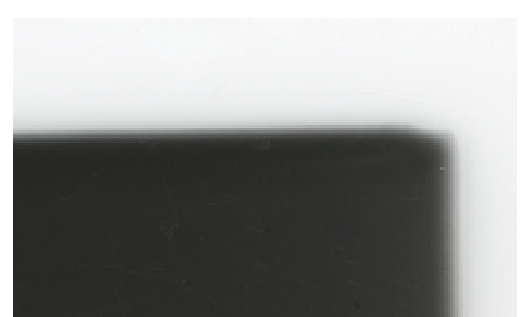

Fig. 1. The ink containing a mixture of cyan, magenta and yellow pigments (dark area) deposited on polycarbonate substrate (white area).

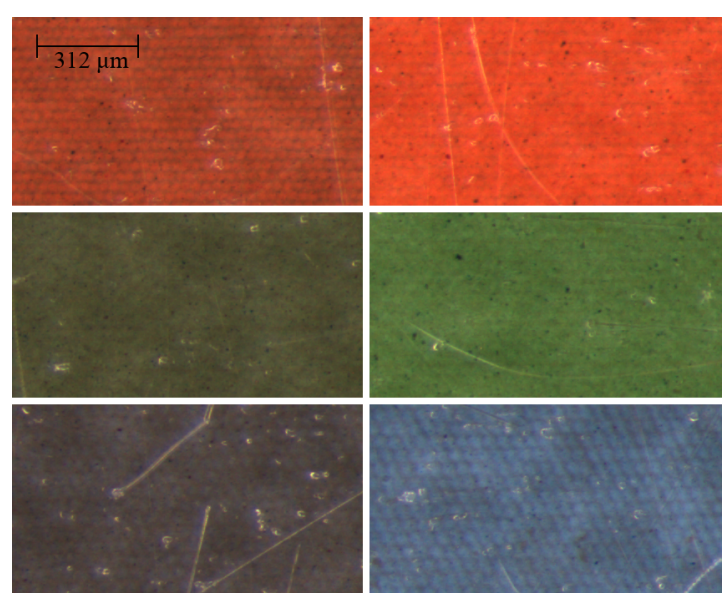

Fig. 2. Microscopic images of areas printed in red, green, blue obtained by irradiation with, respectively, red laser, green laser and blue laser at relative power 0.5 (left) or 1 (right). The coloring layer is nearly continuous, no halftoning is needed. Higher laser power gives brighter color.

The purely physical aspects of color are characterized by the means of the reflectance/absorption spectroscopy. Within organic dyestuffs, the observed relatively narrow absorption bands in the visible region correspond to their lowest electronic excitation energies and are most often explained in terms of the molecular orbital (MO) approach [8]. Empirically, the relatively low excitation energy is observed when the corresponding molecule includes a chain of conjugated double bonds connecting donor and acceptor moieties. The light-induced excitation (HOMO-LUMO electronic transition) is of the charge transfer type, and results in redistribution of electron density along the conjugation chain [9]. For organic pigments (as compared to dyes), another major factor affecting the initial color is the energy of the crystal lattice and intermolecular interactions.

The idea of creating colored images in a pre-deposited pigment mixture consists in trying to selectively bleach the cyan, magenta and yellow colorants by applying strong monochromatic sources of light (such as lasers) of the wavelengths corresponding to their absorption maxima. Electronic excitation of the colorant molecules can give rise to photochemical transformations breaking the bond conjugation and thus leading to the disappearance of the corresponding absorption band. This is the common mechanism of the general color fading (nonselective pigment bleaching) observed under sunlight for textiles or museum paintings [10]. The basic requirement to the choice of colorants for CLM printing is the possibility to bleach them selectively in a mixture. In practice, however, selective addressing is difficult owing to overlapping absorption bands, and even if bleaching takes places, the remaining molecular fragments are not completely colorless. Most often, the residual absorbance is not limited to the near UV region, but exhibits broad bands, infringing into the visible, which results in a dull yellowish tint, sometimes intensified by the degradation of the medium.

Using dye solutions as the primary colored components has some advantages, namely better mastering the selectivity owing to the presence of narrower absorption bands, and lower required light energies for monomolecular reactions. Among existing common applications of selectively addressing organic dyes by the light of the corresponding wavelengths, dye sensitizers for silver-salt based color photography [11], or in photodynamic therapy of cancer [12] can be mentioned. Another example is presented by Lumejet company in Ref. [13]. For security applications in polycarbonate cards, pigments are preferable over dyes, due to their higher aging resistance. Pigments are colorants that are applied into a medium by a dispersion process and remain as solid particles held in place mechanically, usually in a matrix of a solid polymer [14]. They are characterized by high energies of intermolecular bonding (crystalline lattice), and their observed color depends on both light absorption and light scattering components, affected also by their crystalline modification and physical conditioning. Thus, exciting pigment molecules compared to similar dyes requires generally much higher energies (such as those reached by monochromatic laser sources), broadens the corresponding spectral absorption bands, which complicates selective addressing of the colored components, and generates a significant amount of heat that can drastically decrease the reaction selectivity in terms of the product formation, or cause degradation/burning of the surrounding materials.

Calibrating a printing system means establishing the look-up table from RGB values of the original digital layout and the command parameters for the three lasers yielding the targeted printed colors. For that purpose, the exhaustive method consists in varying incrementally all the laser command parameters, measuring the obtained colors with a spectrocolorimeter, and creating an ICC profile. As an advantage, this method accounts for all optical, chemical, thermal, mechanical phenomena whose influence on the printed color is complex and strongly non-linear. However, it is tedious and time consuming, and needs to be repeated every time when any component within the system is modified, including slight variations in the pigment ratio. Instead, we want to print a small selection of colors, and then be able to predict all other ones as it is now permitted for traditional halftone prints (as offset or inkjet) by the last generation of spectral reflectance prediction models [15].

However, these models are not directly transposable to the CLM printing because there is no halftone. From an optical point of view, a card printed by CLM has comparable structure to a card printed by Dye Diffusion Thermal Transfer (D2T2), with a white, opaque layer in the center of the card and, on the top of it, nonscattering films with similar optical indices which can be considered as one absorbing layer. The model proposed by Berns et al. for D2T2 prints [16] will therefore be explored with CLM prints, as it relies on the spectral reflectance of the white opaque layer, the spectral transmittance of the coloring layer and the Fresnel reflectance of the air-overlay interface. In D2T2, for every printable color, the spectral transmittance of the coloring layer can be related to the spectral transmittances of the different dyes and their respective optical thicknesses according to Beer-LambertBouguer law [17]. We will show that for CLM, the assumption that the coloring layer remains a mixture of the same three primary colorants is not satisfied after laser irradiation. In order to obtain a set of primaries and determine, for each color patch, their respective spectral transmittances and optical thicknesses, we thus propose to use 
learning methods from the measurement of a large but reasonable set of printed colors. A Principal Component Analysis (PCA) provides the spectral transmittances of "virtual" primaries. Then a non-linear regression enables linking their respective optical thicknesses to the input RGB values. The comparison between D2T2 and CLM printing will be developed in detail in this study because it seems important to show which elements of Bern's model, known to provide accurate predictions in D2T2, are still valid for the CLM, and which ones need to be revised.

\section{OPTICAL EQUATIONS}

The optical equation predicting the spectral reflectance of a diffusing background in optical contact with a nonscattering coloring layer on the top of it was introduced by Williams and Clapper for photograph prints [18]. Since both the D2T2 and CLM printed cards have this structure, the Williams-Clapper equation applies. It is derived from a description of the multiple reflections of light between the diffusing background and the air-print interface through the coloring layer. The nonscattering layers and the diffusing background are assumed to have same refractive index $n$. By denoting as $\rho(\lambda)$ the internal reflectance of the background, and $t(\lambda)$ the internal transmittance of the coloring layer under normal incidence, the spectral reflectance factor of the print, illuminated at angle $\theta_{i}$ and observed at angle $\theta_{r}$ $\left(\theta_{i}: \theta_{r}\right.$ geometry) is given by

$$
R(\lambda)=r_{s}+\frac{T_{\text {in }} T_{\text {out }} \rho(\lambda) t^{1 / \cos \left(\theta_{i}^{\prime}\right)+1 / \cos \left(\theta_{r}^{\prime}\right)}}{1-r_{i}(t, \lambda) \rho(\lambda)}
$$

where $\theta_{i}^{\prime}$ denotes the orientation of the incident beam after refraction in the coloring layer, and $\theta_{r}^{\prime}$ the orientation of the exiting beam before refraction in air:

$$
\begin{aligned}
& \theta_{i}^{\prime}=\arcsin \left(\sin \left(\theta_{i}\right) / n\right), \\
& \theta_{r}^{\prime}=\arcsin \left(\sin \left(\theta_{r}\right) / n\right)
\end{aligned}
$$

$r_{s}$ is the specularly reflected light component captured by the observer (which is zero if $\theta_{i} \neq \theta_{r}$ ). $T_{\text {in }}$ denotes the Fresnel transmittance of the print-air interface for the incident light, i.e., using same notations as in Ref. [19] for the Fresnel factors:

$$
T_{\text {in }}=T_{01}\left(\theta_{i}\right),
$$

$T_{\text {out }}$ is the Fresnel transmittance of the interface for the exiting light accounting for the change of geometrical extent due to the refraction in air,

$$
T_{\text {out }}=\frac{T_{10}\left(\theta_{r}^{\prime}\right)}{n^{2}}=\frac{T_{01}\left(\theta_{r}\right)}{n^{2}},
$$

and $r_{i}(t, \lambda)$ is the spectral reflectance of the diffuse light that is internally reflected by the interface through the coloring layer:

$$
r_{i}(t, \lambda)=\int_{\theta=0}^{\pi / 2} t^{2 / \cos \theta}(\lambda) R_{10}(\theta) \sin (2 \theta) d \theta
$$

A simplified version of the Williams-Clapper equation is to consider that the coloring layers have an effective internal transmittance $T(\lambda)$ independent of the orientation and angular distribution of light. The Clapper-Yule reflectance model for halftone prints [20], as well as Bern's reflectance model [16] for contone prints rely on this « nonorientational » internal transmittance which is often suitable when the colorant is slightly scattering. The Williams-Clapper equation thus simplifies into the following equation, equivalent to a Saunderson correction [21] for colored diffusing background of reflectance $\rho(\lambda) T^{2}(\lambda)$ (see Fig. 3):

$$
R(\lambda)=r_{s}+\frac{T_{\text {in }} T_{\text {out }} \rho(\lambda) T^{2}(\lambda)}{1-r_{i} \rho(\lambda) T^{2}(\lambda)},
$$

with

$$
r_{i}=\int_{\theta=0}^{\pi / 2} R_{10}(\theta) \sin (2 \theta) d \theta
$$

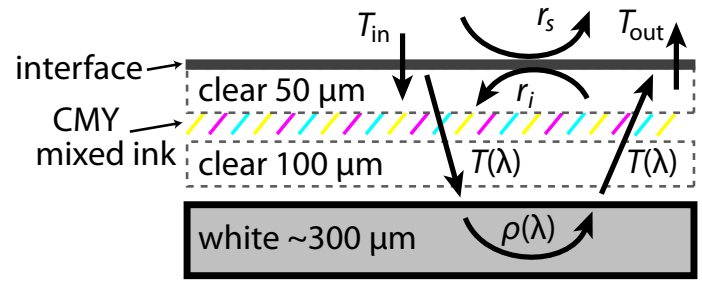

Fig. 3. An example of layer structure of a polycarbonate card for CLM printing.

The refractive index is generally assumed to be $n=1.5$. For a reflectance factor based on the $45^{\circ}: 0^{\circ}$ geometry, we have $T_{i n}=0.95$, $T_{\text {out }}=0.96 / 1.5^{2}, r_{s}=0$, and $r_{i}=0.6$ that are constants attached to the reflections and transmission of light by the air-print interface depending only on the refractive index of the layers [22].

According to Beer-Lambert-Bouger law [17], doubling the thickness of the coloring layer or doubling the concentration in the absorbing substance is equivalent [23]: the internal transmittance $t_{i}(\lambda)$ of the original layer becomes $t_{i}^{2}(\lambda)$, the factor 2 being the relative optical thickness of the coloring substance. In the case where the coloring layer contains a mixture of three colorants, e.g. cyan, magenta and yellow, with respective relative optical thicknesses $\varepsilon_{c}, \varepsilon_{m}$ and $\varepsilon_{y}$, the internal transmittance of the coloring layer is

$$
T\left(\lambda, \varepsilon_{c}, \varepsilon_{m}, \varepsilon_{y}\right)=t_{c}^{\varepsilon_{c}}(\lambda) t_{m}^{\varepsilon_{m}}(\lambda) t_{y}^{\varepsilon_{y}}(\lambda)
$$

and the reflectance of the print, according to Berns' model, becomes:

$$
R\left(\lambda, \varepsilon_{c}, \varepsilon_{m}, \varepsilon_{y}\right)=r_{s}+\frac{T_{i n} T_{\text {out }} \rho(\lambda) t_{c}^{2 \varepsilon_{c}}(\lambda) t_{m}^{2 \varepsilon_{m}}(\lambda) t_{y}^{2 \varepsilon_{y}}(\lambda)}{1-r_{i} \rho(\lambda) t_{c}^{2 \varepsilon_{c}}(\lambda) t_{m}^{2 \varepsilon_{m}}(\lambda) t_{y}^{2 \varepsilon_{y}}(\lambda)}
$$

The diffusing background reflectance $\rho(\lambda)$, and the internal transmittances of the three colorants, $t_{c}(\lambda), t_{m}(\lambda)$ and $t_{y}(\lambda)$, can be deduced from measured data. Since the spectral reflectance factor of the print without ink $(T(\lambda)=1$ ) depends only on $\rho(\lambda)$, we have

$$
R_{0}(\lambda)=r_{s}+\frac{T_{\text {in }} T_{\text {out }} \rho(\lambda)}{1-r_{i} \rho(\lambda)},
$$

therefore,

$$
\rho(\lambda)=\frac{R_{0}(\lambda)-r_{s}}{T_{\text {in }} T_{\text {out }}+r_{i}\left[R_{0}(\lambda)-r_{s}\right]} .
$$

Then, the spectral reflectance factor of the card with a layer of colorant $j$, with internal transmittance $t_{j}(\lambda)$ and unit optical thickness, is given by

$$
R_{j}(\lambda)=r_{s}+\frac{T_{\text {in }} T_{\text {out }} \rho(\lambda) t_{j}^{2}(\lambda)}{1-r_{i} \rho(\lambda) t_{j}^{2}(\lambda)} .
$$

Knowing $R_{j}(\lambda)$ and $\rho(\lambda)$, we can deduce the colorant's internal transmittance

$$
t_{j}(\lambda)=\sqrt{\frac{1}{\rho(\lambda)} \cdot \frac{R_{j}(\lambda)-r_{s}}{T_{\text {in }} T_{\text {out }}+r_{i}\left[R_{j}(\lambda)-r_{s}\right]}} .
$$

This can be repeated for the three colorants. The spectral reflectance of the diffusing background as well as the one of the three colorants on the top of the same diffusing background in the two printing techniques are plotted in Fig. 4. 


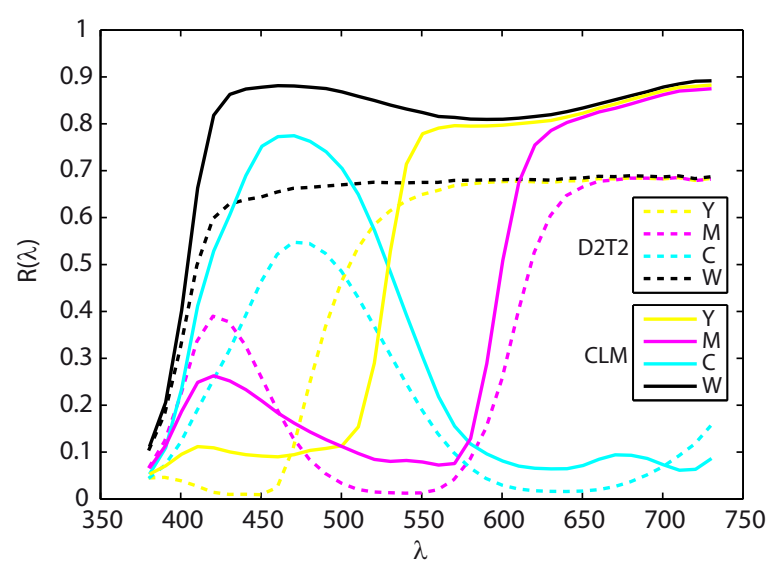

Fig. 4. Measured spectral reflectances of unprinted multilayer polycarbonate card (white background, denoted $\mathrm{W}$ ), and of same substrate with cyan (C), magenta (M), or yellow (Y) colorant layer of unit optical thickness, for D2T2 (dashed line) and the CLM (solid line) printing systems.

\section{APPLICATION OF BERNS' EQUATION TO D2T2- AND CLM-PRINTED CARDS}

In this Section, the goal is to verify if the model expressed by Eq. (9) is suitable for predicting reflectances of laser-bleached colorants. The spectral reflectance of color patches in which the (nonscattering) coloring layer is a mixture of the three colorants with the respective optical thicknesses $\varepsilon_{c}, \varepsilon_{m}$ and $\varepsilon_{y}$ is given by Eq. (9). Usually, these optical thicknesses are not precisely known. For example, in D2T2 printing, color corrections by the driver and the back diffusion effect [16] are not precisely known. That is why a calibration of the model is necessary to get the effective optical thicknesses corresponding to the amount of dyes actually present on the support, which may significantly differ from the nominal ones. The first question is to know whether they can be deduced from the measured spectral reflectances of the color patches, and whether the predicted reflectances with these obtained optical thicknesses correspond to the measured ones. We consider here a coloring layer made of three primary colorants. For a given patch, the relative optical thicknesses of these primary colorants are deduced by using the Levenberg-Marquardt minimization algorithm [24-26] from the following objective function based on the measured reflectance $R_{m}(\lambda)$ and predicted reflectance $R\left(\lambda, \varepsilon_{c}, \varepsilon_{m}, \varepsilon_{y}\right)$ expressed by Eq. (9):

$$
\left(\varepsilon_{c}, \varepsilon_{m}, \varepsilon_{y}\right)=\underset{\hat{\varepsilon}_{c}, \hat{\varepsilon}_{m}, \hat{\varepsilon}_{y}}{\operatorname{argmin}} \sum_{\lambda}\left(R_{m}(\lambda)-R\left(\lambda, \hat{\varepsilon}_{c}, \hat{\varepsilon}_{m}, \hat{\varepsilon}_{y}\right)\right)^{2},
$$

The initial optical thickness values considered in the algorithm are related to the RGB values of the original digital layout, $r, g$ and $b$, expressed in the range $[0,1]$ :

$$
\left(\varepsilon_{c}^{0}, \varepsilon_{m}^{0}, \varepsilon_{y}^{0}\right)=(1-r, 1-g, 1-b)
$$

In the following, in order to assess the agreement between measured and predicted spectra, we will use the equivalent color difference CIELAB 1994 DeltaE metric $\Delta E_{94}$ [17], obtained by converting the predicted and measured spectra into CIE-XYZ tristimulus values, calculated with a D65 illuminant and in respect to the $2^{\circ}$ standard observer, then by converting the CIE-XYZ values into CIELAB color coordinates using as a white reference the white point of the D65 illuminant.

Let us consider the case of CLM. Three white polycarbonate cards coated separately with the three primary pigmented layers (cyan, magenta and yellow) were obtained from the colorant manufacturer. The layer thicknesses and pigment concentrations were not communicated but we attributed a unit optical thickness to each individual pigment layer before bleaching. From the measured spectral reflectances of these samples, we could deduce according to Eq. (13) the spectral internal transmittances of the cyan, magenta and yellow colorants, respectively $t_{c}(\lambda), t_{m}(\lambda), t_{y}(\lambda)$. Then, from the measured spectral reflectance of a card coated with a mixture of the three colorants, we could evaluate the relative optical thicknesses of the cyan, magenta, and yellow colorants, respectively $\varepsilon_{c}=1.026$, $\varepsilon_{m}=0.872$, and $\varepsilon_{y}=0.751$. If the three single-colorant samples provided by the manufacturer have the same layer thickness and the same pigment concentration, the relative optical thicknesses mentioned above would correspond to the proportions of each colorant in the mixture. Fig. 5 shows that the spectral reflectance predicted by Eq. (9) with these $\varepsilon_{j}$ values agrees with the measured one but does not coincide perfectly: the $\Delta E_{94}$ between the two spectra is 1.08 unit, therefore above the just noticeable difference. This might be due to slight variations of pigment conditioning between the single-colorant sample and the colorant mixture coated in the studied cards.

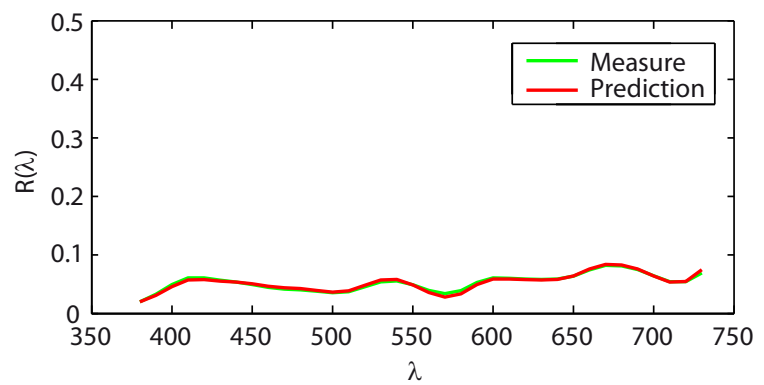

Fig. 5. Predicted and measured spectral reflectances of the nonirradiated colorant mixture on polycarbonate card in CLM with fitted optical thickness values deduced from Eq. (14).
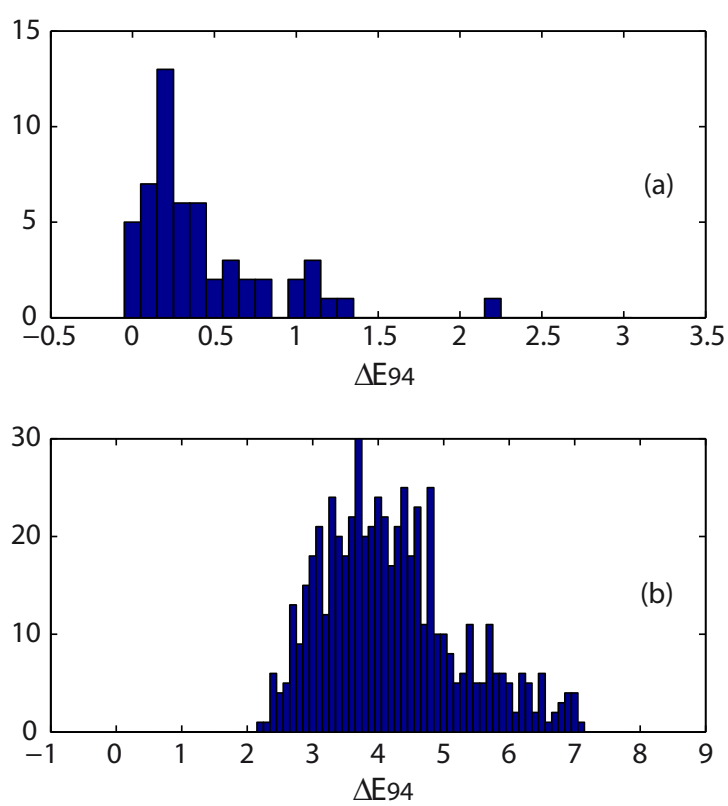

Fig. 6. Distributions of the $\Delta \mathrm{E}_{94}$ values computed between measured and predicted spectral reflectances, given by Berns' equation with fitted optical thickness of the three dyes, a) for D2T2 printed cards and b) with CLM printed cards.

A similar operation was performed with 570 CLM color patches printed on 24 cards using fixed laser setup (wavelengths, focal distance, frequency, min and max powers, gamma correction function, and other specific parameters). For comparison, it was also performed with 54 D2T2 color patches. For each patch, the set of optical thicknesses $\left\{\varepsilon_{c}, \varepsilon_{m}, \varepsilon_{y}\right\}$ was computed according to Eq. (14) by considering the corresponding primaries. The computed $\Delta E_{94}$ between the predicted spectra and the measured ones are displayed as histograms in Fig. 6. 

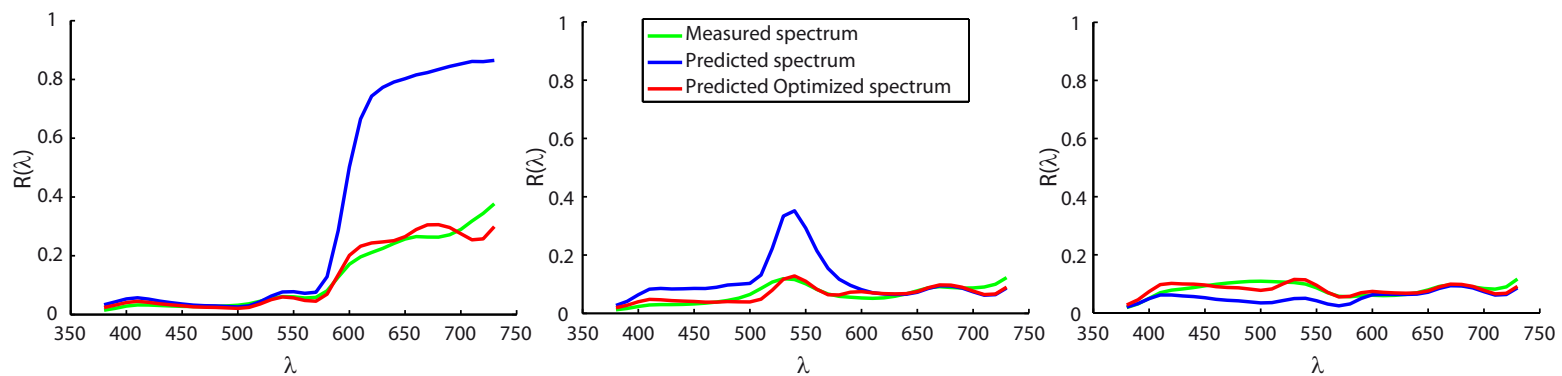

Fig. 7. Measured and predicted spectral reflectances of three CLM printed patches. The predicted spectra in blue line assume that one colorant has an optical thickness 0 and the other two colorants have an optical thickness 1 . Those in red line were obtained with fitted optical thickness of the three colorants.

Regarding the D2T2 printing, the average $\Delta E_{94}$ value of 0.43 unit is low. This shows that once the spectral parameters $\rho(\lambda), t_{c}(\lambda)$, $t_{m}(\lambda)$, and $t_{y}(\lambda)$ are known, the spectral reflectance of any color patch can be accurately predicted once the correct optical thicknesses values $\left\{\varepsilon_{c}, \varepsilon_{m}, \varepsilon_{y}\right\}$ are specified. This is not true in CLM printing for which a high average $\Delta E_{94}$ value of 4.2 units was obtained, with a maximum of 7.1 units. Even by fitting the optimal $\left\{\varepsilon_{c}, \varepsilon_{m}, \varepsilon_{y}\right\}$ values, the predicted spectral reflectances still deviate significantly from the measured ones, which does not enable to validate so far the model for CLM prints.

By way of illustration, we analyzed the "red", "green" and "blue" patches shown in the right column of Fig. 2, obtained by irradiating the card with, respectively, the red laser alone, the green laser alone, and the blue laser alone, at maximal power. Their measured spectral reflectances are plotted in Fig. 7 in green line. With an ideal bleaching process, one colorant would be fully bleached under the laser irradiation, and the two other ones would not be modified. The spectra predicted according to this assumption, plotted in blue line, are very far from the measured ones (green line) especially in the long wavelength domain for the irradiation by the red laser $(660 \mathrm{~nm})$, in the middle wavelength domain for the irradiation by the green laser $(532 \mathrm{~nm})$ and in the short wavelength domain for the irradiation by the blue laser ( $440 \mathrm{~nm}$ ). By fitting the optical thicknesses of the three dyes using Eq. (14), the predicted spectra (red lines) are closer to the measured ones but significant deviation is still observed. We must conclude that the spectral reflectance of these patches cannot be expressed in terms of a combination of three primary transmittances $t_{c}(\lambda), t_{m}(\lambda), t_{y}(\lambda)$, probably because the colorant mixture after irradiation does not contain the same absorbing substances as before irradiation. In addition to targeted photobleaching, laser-induced local increases in temperature may result also in thermal decomposition of all the materials present in the substrate. Thus, apart from the initial colored components, the final image includes dull-colored decomposition products of both the initial pigments and the polymer medium. Their contribution is strongly dependent on the particular materials and the bleaching technique. The conclusion is that the model proposed by Berns and based on three primary colorants with varied optical thicknesses applies for D2T2 printing, but not for CLM printing. Therefore, the next questions are whether we can consider for CLM printing more primary colorants, how many are necessary, and how they can be determined.

Table 2): most of them are close to zero, thus meaning that the corresponding singular vectors (our primaries) can be ignored. Hence, a number $M<N$ of primaries is sufficient to accurately approximate the spectrum, which can therefore be written

$$
S(\lambda)=\sum_{i=1}^{M}\left\langle S(\lambda) \mid S_{i}(\lambda)\right\rangle S_{i}(\lambda)+e(\lambda),
$$

where $e(\lambda)$ is a spectral error that we consider sufficiently small to have no impact on the perceived color.

Two versions of the PCA are permitted according to whether we compute it directly from the spectral reflectances of the patches (in this

\section{APPLICATION OF THE METHOD ON VIRTUAL PRIMARIES OBTAINED BY PCA}

The set of spectral reflectances which can be obtained with a given printing technology forms a vector space whose effective dimension depends on the number of colorants or inks, but also on the support and possibly on optical phenomena taking place within the print. One method to determine the dimensionality of such a spectral space is the Principal Component Analysis (PCA) [27-29], applied on a set of measured spectral reflectances of patches.

Consider discrete spectra containing $N$ values. For instance, $N=36$ for spectra measured between 380 and $730 \mathrm{~nm}$ in steps of $10 \mathrm{~nm}$. Every spectrum $S(\lambda)$ can be written as a weighted sum of $N$ base spectra $S_{i}(\lambda)$, called primaries:

$$
S(\lambda)=\sum_{i=1}^{N} a_{i} S_{i}(\lambda)
$$

where $\left(a_{1}, a_{2}, \ldots, a_{N}\right)$ represent the coordinates of the spectrum in this basis $\left\{S_{1}, S_{2}, \ldots, S_{N}\right\}$ which are the projection of the spectrum $S(\lambda)$ onto the basis $S_{i}(\lambda)$, therefore given by the dot product, denoted $\langle. \mid\rangle$, of $S(\lambda)$ and $S_{i}(\lambda)$ :

$$
S(\lambda)=\sum_{i=1}^{N}\left\langle S(\lambda) \mid S_{i}(\lambda)\right\rangle S_{i}(\lambda)
$$

Alternatively to PCA, we can use the Singular Value Decomposition (SVD) [27, 30]. Let us consider $p$ spectra and form a matrix $\mathbf{P}$ of size $p \times N$ whose rows are the different spectra. The SVD decomposes $\mathbf{P}$ as:

$$
\mathbf{P}=\mathbf{U} \cdot \mathbf{V} \cdot \mathbf{W}^{T}
$$

where $\mathbf{U}$ is a $p \times p$ unitary matrix, $\mathbf{V}$ a $p \times N$ rectangular diagonal matrix with non-negative real diagonal entries (the singular values $\sigma_{i}$ in decreasing order), and $\mathbf{W}$ is a $N \times N$ unitary matrix. The primaries $S_{i}(\lambda)$ correspond to the $N$ columns of matrix $\mathbf{W}$.

In practice, the set of spectra produced by a printing technology is a subset of the $\mathrm{N}$-dimensional space whose dimension is much lower than the number of wavebands in the spectra. This can be easily observed through the singular values (see

case the spectra incorporate the influence of the diffusing support and the multiple reflections of light within the print) or from the coloring layer's internal transmittances deduced according to Eq. (13) from the measured spectral reflectances of the print. Tzeng and Berns [28], following the studies [31] and [32], showed that a PCA performed on the logarithm of internal transmittances of the coloring layers (or, their spectral absorbances, or their optical densities) is more relevant than a PCA performed on measured reflectances of prints, especially when the learning set is small. Furthermore, through the logarithm of the internal transmittances, we can get a linear equation similar to Eq. (19) . The PCA dimensionality reduction method based is therefore made on the logarithm of spectral internal transmittances. 
The $i^{\text {th }}$ column of matrix $\mathbf{W}$ in Eq (18), denoted $W_{i}(\lambda)$, corresponds to the logarithm of the transmittance of the $i^{\text {th }}$ primary, and we thus have

$$
W_{i}(\lambda)=\log \left(t_{i}(\lambda)\right)
$$

or equivalently

$$
t_{i}(\lambda)=\exp \left(W_{i}(\lambda)\right)
$$

For any other patch of internal transmittance $t(\lambda)$, we define:

$$
\left\langle\log (t(\lambda)) \mid W_{i}(\lambda)\right\rangle=\varepsilon_{i},
$$

and we can write

Table 2. We see that the three first ones are much higher than the following ones, which indicates that the dimensionality of spectral space generated by D2T2 printing is 3. This conclusion could also be drawn from the computation of the cumulative percentage variance as it exceeds $99.9 \%$ with three primaries. This is consistent with the fact that three dyes are mixed and that the printing of each ink does not modify the spectral transmittance of the other inks.

The first ten singular values obtained from 130 CLM printed patches are also given in Table 1 . The dimensionality of the spectral space generated by CLM is higher than the one generated by D2T2 since the fourth singular value is higher and still significant. Once again, being given the cumulative percentage variance, the whole space (beyond 99.9\%) is recovered using, this time, four primaries.

The first six virtual primary transmittances, used in Eq. (21) corresponding to the exponential of vectors $W_{i}(\lambda)$ issued from the PCA dimensionality reduction method, are plotted in Fig. 8. As often with PCA, the basis vectors have rather a mathematical than physical meaning: here, the transmittances are higher than 1 . We tested the spectrum reconstruction accuracy with 2 to 10 primaries issued from Eq. (6) and Eq. (21) for the 570 printed colors in CLM, and plotted the average $\Delta E_{94}$ value as a function of the number of primaries (Fig. 9). In order to study the influence of the number of spectra used to compute the SVD on the reconstruction accuracy, we computed it from either 294, 130, 98, 62, or 12 patches. In either case, the reconstruction based on 3 primary spectra is poor (average of 3.3 units), as already noticed in Fig. 6, but it significantly drops from 4 primary spectra, which confirms that CLM generates a 4-dimensional space. It is interesting to notice that beyond 62 patches, the reconstruction accuracy remains unchanged. By refining the approximation with 6 primaries, the average value for $\Delta E_{94}$ over the 570 patches is decreased to 0.10 unit and its maximum to 0.39 unit. This is sufficient to ensure good reconstruction of spectral reflectances of CLM printed colors.

$$
\log [t(\lambda)]=\sum_{i=1}^{M} \varepsilon_{i} \log \left[t_{i}(\lambda)\right]+e(\lambda)
$$

The $M$ primaries with internal transmittances $t_{i}(\lambda)$ form the new set of "virtual" primaries. The internal transmittance of a dye mixture is written in terms of these primary transmittances as:

$$
T(\lambda)=\prod_{i=1}^{M}\left(t_{i}^{\varepsilon_{i}}(\lambda)\right)
$$

and the spectral reflectance of the patch with this mixture is given by Eq. (6). Obviously, only the more significant primaries are to be considered.

According to our experiments carried out with 54 D2T2 printed patches, the first ten singular values in decreasing order, issued from the SVD, are presented in

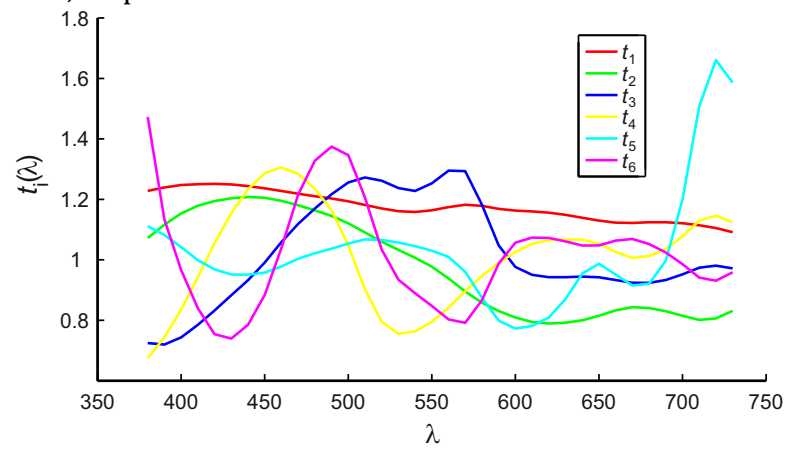

Fig. 8. Virtual primary transmittances corresponding to the exponential of the first six basis vectors issued from the PCA.

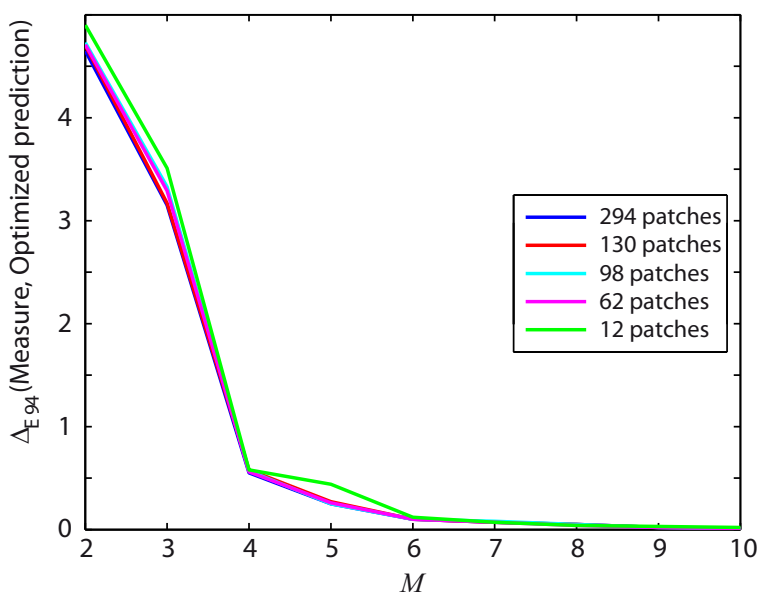

\begin{tabular}{|c|c|c|c|c|c|c|c|c|c|c|}
\hline ith singuar value & 1 & 2 & 3 & 4 & 5 & 6 & 7 & 8 & 9 & 10 \\
\hline$\sigma_{i}$ & 23.00 & 12.10 & 10.30 & 0.35 & 0.33 & 0.17 & 0.10 & 0.09 & 0.05 & 0.04 \\
\hline Variance & 529 & 146.41 & 106.09 & 0.1225 & 0.1089 & 0.0289 & 0.0100 & 0.0081 & 0.0025 & 0.0016 \\
\hline \% Variance & 67.67 & 18.73 & 13.57 & 0.02 & 0.01 & 0 & 0 & 0 & 0 & 0 \\
\hline Cumulative \% variance & 67.666 & 86.394 & 99.964 & 99.98 & 99.993 & 99.997 & 99.998 & 99.999 & 100 & 100 \\
\hline$\sigma_{i}-\sigma_{i+1}$ & 0.83 & 0.53 & 0.47 & 0.70 & 0.10 & 0.67 & 0.17 & 0.27 & 0.36 & - \\
\hline Variance & 2401 & 65.6100 & 14.4400 & 4.0000 & 0.3721 & 0.3025 & 0.0324 & 0.0225 & 0.0121 & 0.0049 \\
\hline$\%$ Variance & 96.59 & 2.64 & 0.58 & 0.16 & 0.01 & 0.01 & 0 & 0 & 0 & 0 \\
\hline Cumulative \% variance & 96.589 & 99.228 & 99.809 & 99.970 & 99.985 & 99.997 & 99.998 & 99.999 & 100 & 100 \\
\hline
\end{tabular}

Fig. 9. Spectral reconstruction accuracy of CLM prints, tested over 570 printed colors, as a function of the number $M$ of primary spectra obtained by SVD computed from various numbers of color patches.

Table 2. Comparison of the dimension of the vector space for D2T2 and CLM printing technologies 


\section{FROM RGB COLOR TO SPECTRAL REFLECTANCE}

With the "virtual" primaries obtained from the SVD in the previous section, we pursue the calibration of the model by associating their respective optical thicknesses in the printed card for every RGB color of the digital layout used as command for the lasers.

Let us denote as $M$ the number of 'virtual' primaries. A third order polynomial regression is used, according to a simplified method presented in Ref. [33], in order to find a transformation $\mathbf{P}$, containing the polynomial regression coefficients, that maps the input $R, G, B$ values to the $M$ optical thicknesses of primaries. At the third order, the matrix $\mathbf{P}$ representing the calibration of the predictive model is a $20 \times M$ matrix:

$$
\mathbf{P}=\left[\begin{array}{cccc}
p_{1}^{\varepsilon_{1}} & p_{1}^{\varepsilon_{2}} & \ldots & p_{1}^{\varepsilon_{M}} \\
p_{2}^{\varepsilon_{1}} & p_{2}^{\varepsilon_{2}} & \ldots & p_{2}^{\varepsilon_{M}} \\
\ldots & \ldots & \ldots & \ldots \\
p_{20}^{\varepsilon_{1}} & p_{20}^{\varepsilon_{2}} & \ldots & p_{20}^{\varepsilon_{M}}
\end{array}\right] .
$$

For a given color patch $j$ originally defined by its RGB values $\left(r_{j}, g_{j}, b_{j}\right)$, converted in CMY values according to Eq (15) $\left(C_{j}, M_{j}, Y_{j}\right)=\left(1-r_{j}, 1-g_{j}, 1-b_{j}\right)$, we define the following 20 dimensional vector

$$
\begin{gathered}
\mathbf{d}_{j}=\left(1, C_{j}, M_{j}, Y_{j}, C_{j}^{2}, C_{j} M_{j}, C_{j} Y_{j}, M_{j}^{2}, Y_{j}^{2}, M_{j} Y_{j}, C_{j}^{2} M_{j},\right. \\
\left.C_{j}^{2} Y_{j}, C_{j}^{3}, C_{j} M_{j}^{2}, C_{j} Y_{j}^{2}, C_{j} M_{j} Y_{j}, M_{j}^{2} Y_{j}, M_{j} Y_{j}^{2}, M_{j}^{3}, Y_{j}^{3}\right)
\end{gathered}
$$

and then obtain the set of optical thicknesses $\boldsymbol{\varepsilon}_{j}^{c a l}=\left(\varepsilon_{1, j}^{c a l}, \varepsilon_{2, j}^{c a l}, \ldots, \varepsilon_{M, j}^{c a l}\right)$ (representing the $M$ calibrated input parameters to predict patch $j$ ) from the following matrix product:

$$
\boldsymbol{\varepsilon}_{j}^{c a l}=\mathbf{d}_{j} \cdot \mathbf{P} .
$$

The $20 \times M$ entries of the matrix $\mathbf{P}$ are obtained from a learning set of $N$ printed color patches, by solving the following matrix equation in the least-mean square sense by using for example the operator \or the mldivide function with Matlab:

$$
\left[\begin{array}{c}
\boldsymbol{\varepsilon}_{1}^{\text {projected }} \\
\boldsymbol{\varepsilon}_{2}^{\text {projected }} \\
\ldots \\
\boldsymbol{\varepsilon}_{N}^{\text {projected }}
\end{array}\right] \equiv\left[\begin{array}{c}
\mathbf{d}_{1} \\
\mathbf{d}_{2} \\
\cdots \\
\mathbf{d}_{N}
\end{array}\right] \cdot \mathbf{P}
$$

where each row of the matrices bordering the $\equiv$ symbol is a line vector attached to one of the $N$ color patches of the calibration set. For a calibration patch $i$, the optical thicknesses vectors $\left(\varepsilon_{1, i}^{\text {projected }}, \varepsilon_{2, i}^{\text {projected }}, \ldots, \varepsilon_{M, i}^{\text {projected }}\right)^{\prime}$ are obtained according to the method presented in the previous section, with Eq. (20) by ignoring $e(\lambda)$, for a set of $N$ patches $(N \geq 20)$. The vectors $\mathbf{d}_{i}(i=1 . . N)$ are given by Eq. (23).

The capacity of the three-order regression represented by $\mathbf{P}$ to provide accurate optical thickness values for every input RGB values depends on the smoothness of relationship between these input and output values. When small variations of the RGB values induce strong variations of the optical thickness values, the accuracy of the method might be poor. However, it is possible to split the RGB space into several subspaces in which the variations of optical thicknesses are more reasonable, and to define a matrix $\mathbf{P}$ in each subspace. A similar idea was proposed by Zhang, not for the computation of a transformation matrix but for the computation of the PCA [34]]. In our experiments with CLM, we observed that the RGB-to-opticalthicknesses (RGB-to-E) transform is very different according to the power of the blue laser (denoted as $b$ ). We therefore splitted the RGB space into two subspaces: a first one corresponding to the low power range of the blue laser $(b \in \llbracket 0,85 \rrbracket$ on 8 bits in our experiments), and a second one to the high power range of this laser $(b \in \llbracket 86,255 \rrbracket$ on 8 bits). The influence of blue laser power on color gradients is illustrated in Fig. 10 by a representation of the corresponding colors in the CIELAB $\left(a^{*}, b^{*}\right)$-plane: with low blue laser power, more saturated but darker colors are obtained, whereas with high blue laser power, higher luminance is achieved, but chroma is lower and strong color drifts are observed. It would be possible to split the RGB space into more than two subspaces, but more patches would be needed to calibrate the model in each subspace. The two subspaces described above seemed optimal in our application.

Two learning sets were selected in these two RGB subspaces, and we obtained two transformation matrices $\mathbf{P}_{1}$ and $\mathbf{P}_{2}$. For each transformation, $N=65$ appeared a good tradeoff between the number of patches to print, and the performances. Actually, these two sets of 65 patches are those constituting the set of 130 patches used to compute the SVD in the previous section. The same basis vectors issued from these 130 patches are used for the two sub-spaces.

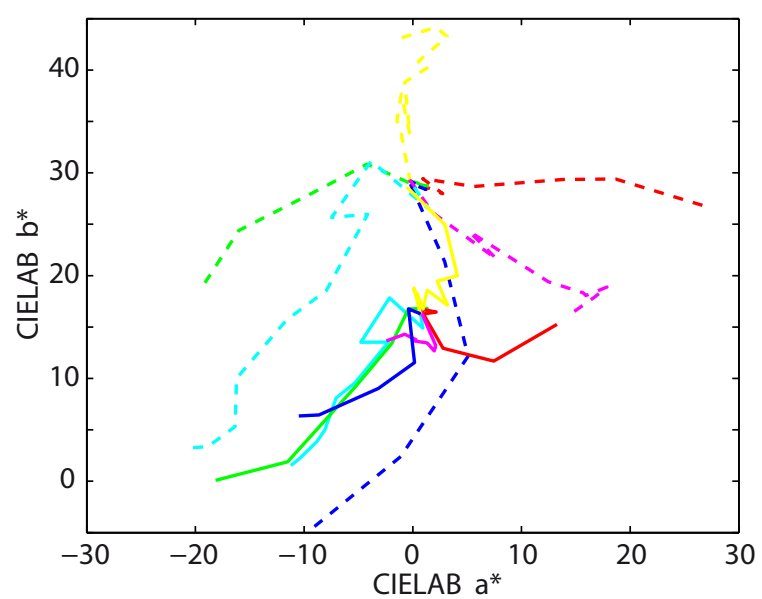

Fig. 10. Red, Green, Blue, Yellow, Magenta, and Cyan color gradients obtained with low (dashed line) and high (solid line) blue laser power.

It seems important to discuss the selection of the patches to print for the calibration of the model for the important impact it may have on the final prediction accuracy. It is possible to select them manually by following the intuitive idea that good learning is achieved when they are well distributed over the RGB subspace, a usual approach in traditional CMYK printing [료] or in applications where odd chips patches in Munsell Atlas are used for learning [34]. However, due to strong non-linearity of the color variations as the laser powers increase (see Fig. 10), it looks better to make the selection in the output CIELAB color space. Indeed, in the brightest part of the gamut, slight power variations of lasers can induce strong color variations, certainly reproducible, but difficult to render correctly with a polynomial regression. We represented in the CIELAB $\left(a^{*}, b^{*}\right)$ plane all the colors that we printed. First, we arbitrarily subtracted a subset of them which will be used for testing. Among the remaining patches, we manually selected the set of patches for learning by including those with highest saturation, and those located in the border of the gamut volume, especially the patches contained in the red, green, and blue gradient wedges. Alternatively, we can randomly select the learning patches with an automated routine. By way of illustration, Fig. 11 gives an overview of the printed colors obtained in the subset corresponding to the low blue laser power. We applied several times the automated selection routine in order to verify that the similar prediction accuracy is achieved at every run of the routine. Indeed, the average CIELAB $\Delta E_{94}$ value computed 40 times between the predicted colors of the testing set and the measured ones was 1.27 unit with a standard deviation of only $9.39 \cdot 10^{-2}$ unit. Note that the color gamut is not centered on the grey level axis, i.e. the point $(0,0)$ in the $\left(a^{*}, b^{*}\right)$ plane. We might expect that further development of the technology could achieve a more centered gamut. 


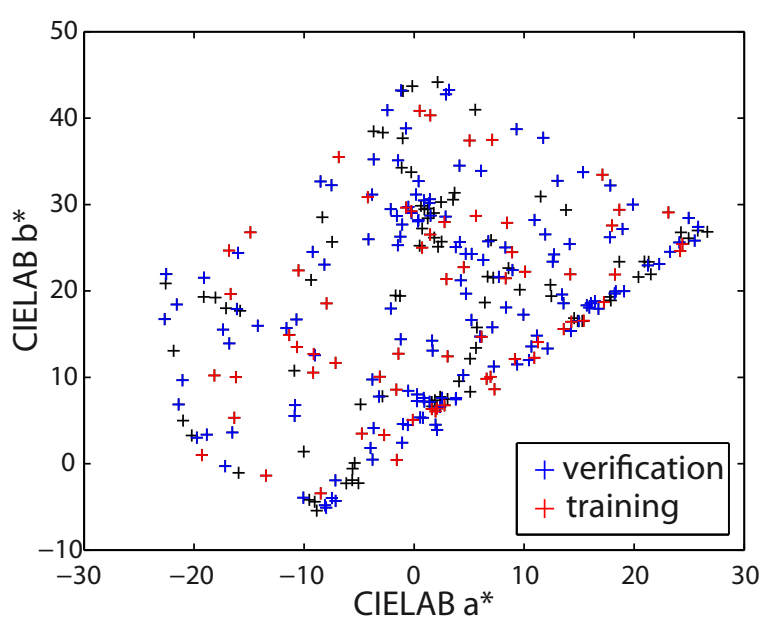

Fig. 11. Printed patches colors represented in the CIELAB $\left(a^{*}, b^{*}\right)$ plane, among which those used for the learning of the RGB to optical thicknesses transform, and for the verification of the model.

\section{PERFORMANCES \& ACCURACY TESTING}

A verification set containing 276 CLM-printed patches, different from the ones used for learning was selected (this time corresponding to colors in the whole RGB space) in order to evaluate the global performance of the calibration, including the determination by SVD of the 6 more relevant primaries and the computation of their respective optimal thicknesses by using the transformation matrix $\mathbf{P}_{1}$, or accordingly $\mathbf{P}_{2}$ with overall 130 learning patches selected by our automatic routine. The histogram of the CIELAB $\Delta E_{94}$ values computed between the measured and predicted spectra is displayed in Fig. 12. We obtained an average $\Delta E_{94}$ value of 1.18 unit, and a maximum of 3.13 units, which is a fairly good accuracy for this strongly non-linear color printing system. The difference between this score of 1.18 unit and the one of 0.10 unit mentioned in Section 4 corresponds to the imprecision of the matrix transformations $\mathbf{P}_{1}$ and $\mathbf{P}_{2}$ relating the RGB input laser intensities with the optical thicknesses of the virtual primaries. In comparison to the model based on Berns' equation presented in Section 3, the gain in prediction accuracy, which clearly appears by comparing the histograms of Fig. 6 (b) and Fig. 12, is remarkable.

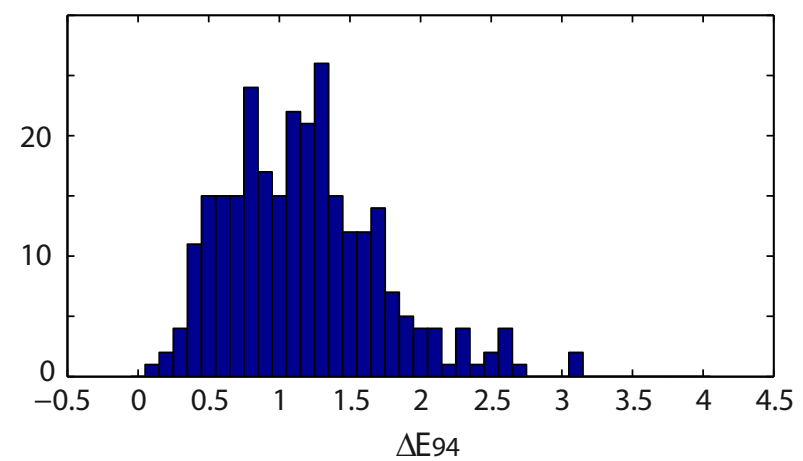

Fig. 12. Histogram representing the distribution of $\Delta \mathrm{E}_{94}$ values computed from the measured reflectance of the 276 CLM verification patches and the reflectance predicted by our method.

The variations of the prediction accuracy (i.e., the average CIELAB $\Delta E_{94}$ value over the testing patch set) according to the number of primaries selected after the PCA and the number of learning patches used for the calibration of the model were assessed. The curves plotted in Figure 13 indicate that at least 4 primaries are necessary, but more than 4 primaries do not improve much the prediction. This is true whatever is the number of learning patches. This is probably due to the fact that the RGB-to-E transform, which maps the 3-dimensional RGB space onto the $M$-dimensional optical thicknesses space, is more imprecise when $M$ increases, thus compensating the gain in prediction accuracy observed in Section 1 with up to 6 primaries.

Regarding the number of learning patches, increasing them improves the global accuracy but to a rather small extent: using 394 patches (197 in each RGB subspace) instead of 130 decreases the average $\Delta E_{94}$ value by only 0.15 unit. Using 62 patches (31 in each RGB subspace) increases it by 0.5 unit, which is a significant loss of accuracy, but still acceptable if the number of calibration patches needs to be limited. 130 patches is rather reasonable compared to the 44 color patches required for the calibration of classic predictive models for traditional ink-based printer, such as the Clapper-Yule or the YuleNielsen modified Spectral Neugebauer models, which are easier to calibrate [15]. The prediction accuracy is also satisfying in comparison with traditional printing. This is noticeable as the CLM printing system that we used (including the ink coating system and the laser marking) is a prototype in development (which has not reached yet the optimal reliability and reproducibility of an industrial system).

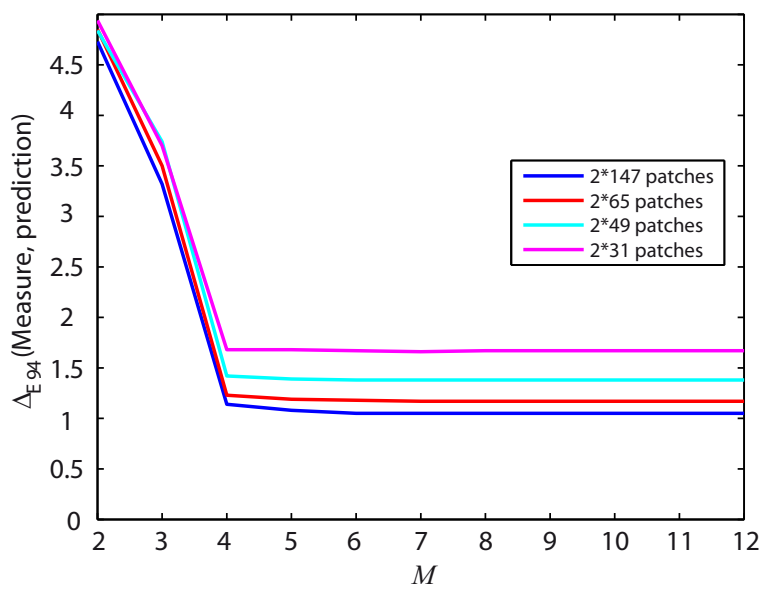

Figure 13. Performances of the prediction according to the number of learning patches and the number of considered primaries.

\section{CONCLUSION}

The CLM printing system illustrates the issue of using a spectral reflectance prediction method developed for an ink-transfer printing system with surfaces printed with a laser-induced coloration technique, even when the optical equation underlying the prediction model seems adapted to these surfaces. In contrast with ink-transfer printing where the spectral properties and amounts of transferred inks can be easily deduced from a few specific printed patches, the spectral properties and amounts of coloring components are different for each combination of power settings of the three lasers and do not seem to follow simple laws. Hence, existing spectral reflectance prediction methods need several adaptations. Firstly, in contrast with halftone printing, the CLM can produce a variety of colors in each printable pixel and is therefore continuous tone printing system. The amounts of primary colorants in the coloring layer are thus expressed in terms of optical thicknesses instead of ink surface coverages. Secondly, the modification of the colorants under laser irradiation, depending on the laser power, implies that the spectral transmittances of primary colorants contained in a given printed color patch are not precisely known, whereas in traditional printing, the inks' internal transmittances (each one being possibly raised to a power epsilon) are independent of the amount of other deposited inks. Thirdly, in contrast with halftoning where the amount of each ink can be easily related to the CMYK channels of the digital layout (even in case of ink spreading or mechanical dot gain), this relation is more difficult to establish in CLM printing.

These conceptual differences led us to develop a new spectral reflectance prediction model combining an optical modelization and learning methods: Berns' equation developed for continuous tone 
prints (D2T2) was used with spectral transmittances of "virtual" primary colorants obtained by performing a principal component analysis on a set of spectral reflectances measured on printed patches. The correspondence between the input digital RGB colors and the optical thicknesses of these primaries is obtained by computing a transformation matrix from the same set of measured spectral reflectances. Despite the strong non-linearity of the relationship between the input and output color values, a rather good prediction accuracy was achieved with 130 printed patches for the calibration of the model. This number of patches is rather acceptable if we compare it to the 44 calibration patches required by the most performing spectral reflectance prediction models available today for classic printing systems [15].

\section{FUNDING}

This work was performed within the framework of the LABEX MANUTECH-SISE (ANR-10-LABX-0075) of Université de Lyon, within the program "Investissements d'Avenir" (ANR- 11-IDEX-0007) operated by the French National Research Agency (ANR).

\section{ACKNOWLEDGMENTS}

The authors would like to thank Mr. Joseph Leibenguth, from Gemalto, and Prof. Vladimir Khodorkovsky, from CINaM, CNRS UMR 7325, for interesting discussions throughout this study.

\section{REFERENCES}

1. H. Kipphan, Handbook of Print Media (Springer Ed., 2001).

2. N. Destouches, N. Crespo-Monteiro, L. Nadar, T. Epicier, Y. Liu, Y. Lefkir, and F. Vocanson, "Laser control of metallic nanoparticles for reversible or permanent color marking," in International Conference on Small Science (Orlando, United States, 2012).

3. N. Destouches, J. Martínez-García, M. Hébert, N. Crespo-Monteiro, G. Vitrant, Z. Liu, A. Trémeau, F. Vocanson, F. Pigeon, S. Reynaud, and Y. Lefkir, "Dichroic colored luster of laser-induced silver nanoparticle gratings buried in dense inorganic films," J. Opt. Soc. Am. B 31, C1-C7 (2014).

4. N. Lutz, and G. Zinner, "Plastic body, which is provided in the form of a film, for example, a transfer film or laminate film or which is provided with a film of this type, and method for producing color image on or in a plastic body of this type," (2004), US Patent 2004/0043308 A1.

5. Y. Bielek, and E. Barker, "Laser activated thermochromic compositions," (FLEXCON COMPANY, INC., 2006), WO 2006/113778 A2.

6. L. Fannasch, M. Hennemeyer-Schwenkner, M. Schumacher, and D. Fischer, "Method for the recording of data on/in data supports by means of laser radiation and data supports produced thus," (Orga Kartensysteme, 2006), US 6986926 B2.

7. T. Sakagami, A. Hashimoto, and Y. TERAJl, "Multicolor-development laser marking sheet for card, and laser marking method," (Japan Coloring Co., Ltd., 2013), WO 2013031399 A1.

8. K. Nassau, Color for science, Art and technology (Elsevier, 1998).

9. J. Fabian, and H. Hartmann, Light Absorption of Organic Colorants: Theoretical Treatment and Empirical Rules (Springer-Verlag Berlin Heidelberg, 1980).

10. R. Johnston-Feller, Color science in the examination of museum objects: Nondestructive procedures (Getty Publications, 2001).

11. G. T. Eaton, Photographic Chemistry: In Black-And-White and Color Photography (3rd Ed. - Morgan \& Morgan Inc., 1980).

12. H. Zollinger, Color Chemistry: Syntheses, Properties and Applications of Organic Dyes and Pigments (Wiley-VCH, 2003).

13. LumeJet, "Photographic Quality Digital Printing," http://www.lumejet.com/technology/photographic-quality-digitalprinting.

14. R. M. Christie, Colour Chemistry (The Royal Society of Chemistry, 2001).

15. M. Hébert, and R. Hersch, D., "Review of spectral reflectance models for halftone prints: Principles, Calibration, and prediction accuracy," Color Res. Appl. 40, 383-397 (2015).
16. R. S. Berns, "Spectral modeling of a dye diffusion thermal transfer printer," J. Electron. Imaging 2, 359-370 (1993).

17. G. Wyszecki, and W. S. Stiles, Color Science: Concepts and Methods, Quantitative Data and Formulae, 2nd ed. (John Wiley and Sons, New York, 1982).

18. F. C. Williams, and F. R. Clapper, "Multiple Internal Reflections in Photographic Color Prints," J. Opt. Soc. Am. 43, 595-597 (1953).

19. L. Simonot, M. Hébert, and R. D. Hersch, "Extension of the WilliamsClapper model to stacked nondiffusing colored coatings with different refractive indices," J. Opt. Soc. Am. A 23, 1432-1441 (2006).

20. F. R. Clapper, and J. A. C. Yule, "The Effect of Multiple Internal Reflections on the Densities of Half-tone Prints on Paper," J. Opt. Soc. Am. 43, 600-603 (1953).

21. J. L. Saunderson, "Calculation of the Color of Pigmented Plastics," J. Opt. Soc. Am. 32, 727-729 (1942).

22. D. B. Judd, "Fresnel reflection of diffusely incident light," J. Natl. Bur. Standards 29, 329 (1942).

23. A. D. McNaught, and A. Wilkinson, Compendium of Chemical Terminology (Blackwell Science, Oxford, U.K., 1997).

24. K. Levenberg, "A Method for the Solution of Certain Problems in Least Squares," Quarterly Applied Math. 2, pp. 164-168 (1944).

25. D. W. Marquardt, "An Algorithm for Least-Squares Estimation of Nonlinear Parameters," J. Soc. Indust. Appl. Math. 11, 431-441 (1963).

26. J. J. Moré, "The Levenberg-Marquardt algorithm: Implementation and theory," in Numerical Analysis, G. A. Watson, ed. (Springer Berlin Heidelberg, 1977), pp. 105-116.

27. J. Y. Hardeberg, "On the spectral dimensionality of object colours," Proc. Conference on Colour in Graphics, Imaging, and Vision 2002, 480-485 (2002).

28. D.-Y. Tzeng, and R. S. Berns, "A review of principal component analysis and its applications to color technology," Color Res. Appl. 30, 84-98 (2005).

29. T. Bugnon, "Flexible and Robust Calibration of the Yule-Nielsen Model for CMYK Prints," PhD Dissertation, Ecole Polytechnique Fédérale de Lausanne (2011).

30. W. H. Press, S. A. Teukolsky, W. T. Vetterling, and B. P. Flannery, Numerical recipes in C (2nd ed.): the art of scientific computing (Cambridge University Press, 1992), chap. 2.6.

31. F. H. Imai, R. S. Berns, and D.-Y. Tzeng, "A comparative analysis of spectral reflectance estimated in various spaces using a trichromatic camera system," J. Imag. Sci. Tech. 44, 280-287 (2000).

32. R. S. Berns, "Visible-spectrum imaging techniques: an overview," Proc. SPIE 9th Congress of the International Colour Association 475-480 (2002).

33. R. Charrière, M. Hébert, A. Trémeau, and N. Destouches, "Color calibration of an RGB camera mounted in front of a microscope with strong color distortion," Appl. Opt. 52, 5262-5271 (2013).

34. X. Zhang, and H. Xu, "Reconstructing spectral reflectance by dividing spectral space and extending the principal components in principal component analysis," J. Opt. Soc. Am. A 25, 371-378 (2008). 